\title{
Robust Multi-objective Optimization and Decision for Signal Timing at Single Intersection
}

\author{
Juan Chen \\ SHU-UTS SILC Business School \\ Shanghai University \\ Shanghai, China \\ chenjuan82@shu.edu.cn
}

\author{
Mingxing Jia \\ SHU-UTS SILC Business School \\ Shanghai University \\ Shanghai, China \\ jimmy219@foxmail.com
}

\begin{abstract}
For the purpose of improving the efficiency of traffic signal control with cycle perturbation for single intersections, a robust multi-objective optimization and decision method (RMODM) is proposed. Firstly, this paper presents a non-dominated sorting genetic algorithm II based on the degree of robustness (DR-NSGA-II) to obtain signal timing alternatives. Then, the Minimum deviation Analysis for Subjective and Objective Information (MDASOI) method is proposed to make signal timing decision. The experimental results indicate that the proposed method has preferable control effects.
\end{abstract}

Keywords-multi-objective optimization, robust, decisionmaking, traffic signal control

\section{INTRODUCTION}

The mixed traffic is widely spread in Chinese cities. It is also greatly responsible for major problems, such as conflicts, low efficiency and safety problems. These problems are most evident at street intersections. However, they have not gained enough attention in the past few years. Therefore, research on signal control in the mixed traffic to ensure the harmony between vehicle and slow mode transportation is of great importance.

Intersection signal control is a typical multi-objective problem, and a lot of methods have been developed to handle this problem. Minimizing vehicle delay as the control objective was proposed first by F. Webster in the 1960s, which was called Webster method [1]. Even though the method was developed to minimize a single objective, it presented a widely used approximation formula to calculate average delay in unsaturation flow. In traffic signal control, the sensitivity of traffic performance index to cycle length perturbation is directly related to the overall operating benefits of intersections [2]. Therefore, penetrated study about the traffic signal timing for intersection based on robust multi-objective optimization model with cycle length perturbation have high theoretic and practical values.

First, this paper selects some reasonable control objectives to establish robust multi-objective optimization model, and solves it with an improved genetic algorithm (Non-Dominated Sorting Genetic Algorithm II Based on Degree of Robustness, DR-NSGA-II) to generate robust signal timing Pareto solutions. Then, considering uncertainty preference demand of decisionmakers, the Minimum Deviation Analysis for Subjective and Objective Information (MDASOI) method is proposed to choose the satisfactory signal timing plan.

\section{PROBlem Formulation}

In order to obtain satisfactory control effect, this paper choses average vehicle delay, road capacity, average slow mode transportation delay and vehicle stop rate as the control objectives. These objectives could reflect three important control benefits: The vehicle's benefit is measured by average vehicle delay and road capacity, the pedestrian's benefit by average slow mode transportation delay, and the environmental benefit by vehicle stop rate.

Assuming the traffic flow is uniform flow, we establish a robust multi-objective optimization model with the cycle length perturbation $\delta_{s}^{\prime}$ for intersection signal control, which is described as:

$$
\begin{array}{ll}
\min & F\left(C^{\prime}\right)=\left[f_{1}\left(C^{\prime}\right), f_{2}\left(C^{\prime}\right), f_{3}\left(C^{\prime}\right)\right] \\
\text { s.t. } & C^{\prime}=C+\delta_{s}^{\prime} \quad C_{\min } \leq C \leq C_{\max } \\
& C \lambda_{j} \geq g_{j \min } \quad \alpha \leq 0.9 \\
& j=\{1,2, \cdots, \mathrm{m}\}
\end{array}
$$

Where $f_{1}\left(C^{\prime}\right)$ is the ratio of average vehicle delay $\bar{d}$ to road capacity $Q$ [3], $f_{2}\left(C^{\prime}\right)$ is the average slow mode transportation delay[4], $f_{3}\left(C^{\prime}\right)$ is the vehicle stop rate, $\delta_{s}^{\prime}$ is the cycle length perturbation neighborhood, $C_{\min }, C_{\max }$ are minimum and maximum values of cycle length respectively, $\lambda_{j}$ is the effective green split in the $j$-th phase, $g_{j \min }$ is the minimum green time in the $j$-th phase, $\alpha$ is the degree of saturation of the intersection, $m$ is the number of phases.

\section{Robust Multi-OBJective Optimization Algorithm}

The traffic signal timing control problem, which this paper discusses, is a kind of robust multi-objective optimization problem with a perturbation. Deb et al introduced robustness into the multi objective optimization [5]. Current robust optimization methods usually optimized the mean effective objective functions (instead of the original objective function) computed by averaging a sampling set of neighboring solutions, and always used Monte-Carlo sampling and Latin hypercube sampling (LHS) to achieve better sampling effectivity. However, it is not clear how to determine the sample size.

This paper introduces the degree of robustness to robust multi-objective optimization for obtaining robust optimal 
solutions. An adaptive sampling method is proposed to determine the sample size in the process of computing the degree of robustness for reducing the computation complexity. Note that the current robust multi-objective optimization algorithm does not take a full consideration of the robustness of solutions, we introduce the degree of robustness into the NSGA-II for identifying the set of robust efficient solutions.

\section{A. Mean Effective Function Based On ALHS}

1) Mean Effective Function

Based on the mean effective function [5], in this paper, the robust multi-objective optimization model is described as:

$$
\begin{array}{ll}
\min & F^{e f f}\left(C^{\prime}\right)=\left[f_{1}^{e f f}\left(C^{\prime}\right), f_{2}^{e f f}\left(C^{\prime}\right), f_{3}^{e f f}\left(C^{\prime}\right)\right] \\
\text { s.t. } & C^{\prime}=C+\delta_{s}^{\prime} \quad C_{\min } \leq C \leq C_{\max } \\
& C \lambda_{j} \geq g_{j \min } \quad \alpha \leq 0.9 \\
& j=\{1,2, \cdots, \mathrm{m}\}
\end{array}
$$

Where $f_{l}^{\text {eff }}\left(C^{\prime}\right)$ is the mean effective function described as:

$$
f_{l}^{\text {eff }}\left(C^{\prime}\right)=\frac{1}{\text { Sample }} \sum_{s=1}^{\text {sample }} f_{l}\left(C_{s}^{\prime}\right), l=\{1,2,3\}
$$

Where $C_{s}^{\prime}$ is $s$ - th sample point in the neighborhood of $C$, Sample is the sample size. So the accuracy of mean effective function depends on the reliability of the sampling methods.

\section{2) The calculator of the degree of robustness}

Barrico proposed a new approach to robustness analysis in multi-objective optimization, involving the definition of robustness [6]. Though the utility of the arithmetic to fix the sample size of neighborhood $k \sigma$ improved the calculation accuracy, it also leaded to high computational-complexity. Thus, this paper proposes a new adaptive sampling method to automatically adjust the sample size. The algorithm to determine the sample size in neighborhood $k \sigma$ is described as following:

Step 1: Initialize threshold $\mu$ and $H^{\prime}$ (sample size in neighborhood $\sigma$ ), set $H_{1}=H_{\min }, H_{\max }=H^{\prime}+(k-1) q^{\prime}$

Step 2: Execute LHS, calculate percentage $P_{1}^{\prime}$ of solutions, whose objective function values belong to the neighborhood $\eta$ of $f(X)$, set $m=2, H_{\mathrm{m}}=H_{\max }$

Step 3: Execute LHS, calculate percentage $P_{m}^{\prime}$ of solutions, whose objective function values belong to the neighborhood $\eta$ of $f(X)$

Step 4: If $\left|P_{m}^{\prime}-P_{m-1}^{\prime}\right| \leq \mu$, return Sample ${ }_{k}=\min \left(H_{m}, H_{m-1}\right)$ and the corresponding percentage $P_{m}^{\prime}$, set $P_{k}=P_{m}^{\prime}$, end loop; Else $m=m+1, H_{m}=\left\lfloor\lambda \log \left(\left|\frac{P_{m-1}^{\prime}-P_{m-2}^{\prime}}{\mu}\right|\right)\right\rfloor$, turn to step 3;

Where $H_{\min }, H_{\max }$ are the minimal and maximal number of sample during each iteration respectively, $H_{m}$ is the sample size in $m$-th sample, $\lambda$ is used to adjust the sample size in each iteration, we give $\lambda$ an empirical value of 4 .

When solving mean effective function by evolutionary algorithm, in comparison with the method with fixed sample size (LHS-DR), the total simple size in the above algorithm can be reduced:

$$
\sum_{i=1}^{\text {Gen }} \sum_{j=1}^{N} \sum_{k=1}^{|k|}\left(\text { Sample }_{i, j, k}-\text { Sample }_{i, j, k}^{f}\right)
$$

Where Gen is the number of evolution generations, $N$ is the population size, the Sample $_{i, j, k}$ is sample size of the $j$-th individual in the $i$-th generation, Sample $f_{i, j, k}^{f}$ is the corresponding fixed sample size.

\section{B. NSGA-II Based on Robustness}

Based on the NSGA-II, DR-NSGA-II is proposed, which introduced the degree of robustness into NSGA-II to obtain robust Pareto front. This concept of degree of robustness permits the user to exert a control on the desired level of robustness of the solutions obtain, and to specify the size of the solution neighborhood and the size of sample involved to calculate mean effective function. We suggest maintaining an external population at every generation storing robust elite solutions to ensure that these solutions could take part in the evolution of next generation.

\section{1) Robust External Population}

The robust external population is used to store robust elite solutions, and to conduct the evolution process to obtain the desired robust solutions. Firstly, we compare the degrees of robustness of each solution. The bigger ones are stored in the robust external population. If their degrees of robustness are same, the solutions belonging to the better non-dominated set are chosen. If they also belong to same non-dominated set, the solutions which have longer crowd distance are chosen. Until the size of the robust external population reach the default maximum.

\section{2) Robust partial order}

The partial order defined by Deb is based on the nondominated sorting and crowd distance, but in the problem of robust optimization, the degree of robustness of solutions is an important factor that should be considered. Thus, this paper defines the concept of robust partial order $\left(\prec_{n}\right)$ :

$$
\begin{array}{ll}
\text { - } & i_{\text {rank }}<j_{\text {rank }}, i \prec_{n} j \\
\text { - } & i_{\text {rank }}=j_{\text {rank }} \& i_{r}>j_{r}, i \prec_{n} j \\
\text { - } & i_{\text {rank }}=j_{\text {rank }} \& i_{r}=j_{r} \& i_{d}>j_{d}, i \prec_{n} j
\end{array}
$$

Where $i_{r}, i_{\text {rank }}, i_{d}$ are the degree of robustness, nondomination rank and crowed distance of solution $i$ respectively. Once the individuals are sorted based on the robust partial order, the selection is carried out using the operator $\left(\prec_{n}\right)$. 


\section{InTERVAL Multi-ATtRiBUte DeCision MAKING ANALYSIS OF SignAL TIMING PLANS}

The robust Pareto optimal solutions can be obtained by the above algorithm, while there is still the problem of choosing a particular solution from the Pareto optimal solutions set. We represents the performance index values of the signal timing plan as constant numbers, and the weight of the performance index as interval numbers. Then the problem turns to be an interval multi-attribute decision making (MADM) problem. In the current study of interval MADM, there is little concern about weight assignment of performance index. In addition, though the current posteriori decision guarantee completeness of alternatives set, the computational complexity is high.

Thus, this paper proposes the MDASOI method to reduce the computational complexity. Let $M=\left(\mathrm{M}_{1}, \mathrm{M}_{2}, \cdots \mathrm{M}_{m}\right)$ denotes timing plan set, $D=\left(D_{1}, D_{2}, \cdots D_{n}\right)$ be the performance index, $\omega_{s}=\left(\omega_{s 1}, \omega_{s 2}, \cdots, \omega_{s n}\right)^{T}$ is the subjective interval weight vector, which achieved by expert evaluation. $\omega_{s j}=\left[\omega_{s j}^{l}, \omega_{s j}^{u}\right]$, $\sum_{j=1}^{n} \omega_{s j}^{l} \leq 1, \sum_{j=1}^{n} \omega_{s j}^{u} \geq 1,0 \leq \omega_{s j}^{l} \leq \omega_{s j}^{u} \leq 1$.

\section{A. The construction of signal timing decision matrix}

Let $x_{i j}(\mathrm{i}=1,2, \cdots, m ; j=1,2, \cdots, \mathrm{n})$ be the value of performance index $D_{j}$ of timing plan $M_{i}$, then the decision matrix is described as:

$$
X=\left(\begin{array}{cccc}
x_{11} & x_{12} & \cdots & x_{1 n} \\
x_{21} & x_{22} & \cdots & x_{2 n} \\
\cdots & \cdots & \cdots & \cdots \\
x_{m 1} & x_{m 2} & \cdots & x_{m n}
\end{array}\right)
$$

In MADM problem, the decision matrix allows each attribute to have the same range of measurement. This is achieved by normalizing every element in matrix $X$ into a corresponding element in matrix $V=\left(v_{\mathrm{ij}}\right)_{\mathrm{m} \times \mathrm{n}}$ [7].

\section{B. Integrated Approach to Determine Weights}

In order to determine weights $\bar{\omega}=\left(\overline{\omega_{1}}, \overline{\omega_{2}}, \cdots, \overline{\omega_{n}}\right)^{\mathrm{T}}$ to reflect both subjective consideration of the decision makers and objective information, we minimize the deviation of the subjective information $\omega_{s}$ and objective information $\omega_{0}$. Thus, the weights can be obtained by solving the model:

$$
\operatorname{Min} f(\bar{\omega})=\sum_{j=1}^{n}\left(\frac{\overline{\omega_{j}}-w_{o j}}{w_{o j}}\right)^{2} \text {, s.t. } \omega_{s j}{ }^{l} \leq \overline{\omega_{j}} \leq \omega_{s j}{ }^{u}, \sum_{j=1}^{n} \overline{\omega_{j}}=1
$$

Where the physical interpretation of $f(\bar{\omega})$ is the total deviation between the subjective and objective information, the geometric meaning of which is the square of the distance from a fixed-point in solution space to searching space. Take alternatives with two attribute for example: In Fig. 2, on the horizontal axis is the weight $\omega_{o 1}, \omega_{s 1}{ }^{l}, \omega_{s 1}{ }^{u}$ of one performance index, and on the vertical axis is the weight $\omega_{o 2}, \omega_{s 2}{ }^{l}, \omega_{s 2}{ }^{u}$ of the other performance index. The aim of the (6) is to minimize the distance from the fixed-point $E\left(\omega_{o 1}, \omega_{02}\right)$ to searching space ABCD, which can be divided into nine sections: I, II, III, IV, V, VI, VII, IX .

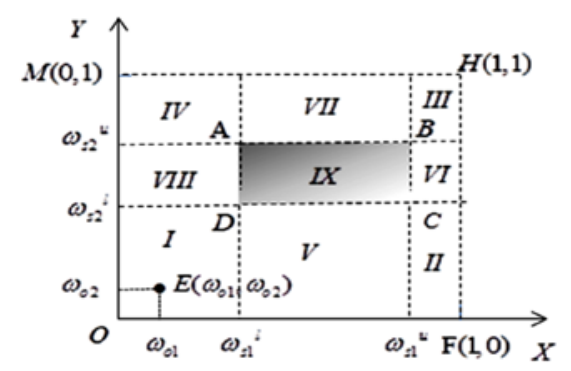

Fig. 1. Optimization model

If there are more than two attributes in the MADM problem, $\bar{\omega}$ is obtained by solving (6) with the utility of simple GA.

\section{Selection of decision plan}

The weighted decision matrix is constructed based on (7), sorted by TOPSIS to obtain the optimal plans.

$$
r_{i j}=\overline{\omega_{j}} \cdot v_{i j}
$$

\section{CASE STUdY}

\section{A. Intersection Data Acquisition}

The intersection of Tianmu West Road and Hengfeng Road in Zhabei district of Shanghai, China is selected to evaluate the proposed optimization and decision method. After intersection channeling design, the signal is controlled by the fixed timing four-phase procedure. According to estimates, the saturation vehicle flow of the main road is $2000 \mathrm{veh} / \mathrm{h}$, which of the secondary road is $1200 \mathrm{veh} / \mathrm{h}$. Be converted to the saturation pedestrians flow, the saturation non-motorized vehicle flow of the main road is $7200 \mathrm{ped} / \mathrm{h}$, the saturation pedestrians flow of the secondary road is $3000 \mathrm{ped} / \mathrm{h}$. In addition, the traffic volume of each entrance during the morning rush hour (8:30 am-9:30 am) is listed in Table I.

TABLE I. EXPERIMENTAL TRAFFIC VOLUME

\begin{tabular}{|c|c|c|c|c|}
\hline & $\begin{array}{c}\text { East } \\
\text { Entrance }\end{array}$ & $\begin{array}{c}\text { West } \\
\text { Entrance }\end{array}$ & $\begin{array}{c}\text { South } \\
\text { Entrance }\end{array}$ & $\begin{array}{c}\text { North } \\
\text { Entrance }\end{array}$ \\
\hline $\begin{array}{c}\text { Volume of } \\
\text { vehicles(veh/h) }\end{array}$ & 1335 & 1461 & 1013 & 814 \\
\hline $\begin{array}{c}\text { Volume of } \\
\text { pedestrians(ped/h) }\end{array}$ & 107 & 94 & 51 & 47 \\
\hline $\begin{array}{c}\text { Volume of } \\
\text { bicycles(bic/h) }\end{array}$ & 921 & 934 & 614 & 620 \\
\hline
\end{tabular}

In this experiment, the cycle length $C$ is from 62 s to $160 \mathrm{~s}$, the minimal green time of every phase is $5 \mathrm{~s}$, and the lost time is 10s. In addition, in the IDR-NSGA-II algorithm, the population size is 100 , the robust external population size is 33 , the maximal evolutionary generation is 300 , the crossover and mutation probability are $0.95,0.05$ respectively, the neighborhood of cycle perturbations is $5 \leq k \delta \leq 30$, where 
$\delta=5$, the degree of robustness $k \in\{1,2,3,4,5,6\}$, the specified percentage parameter $Q=0.75$, the neighborhood of objective function $\eta=\{0.5,0.5,0.5\}$

\section{B. The efficiency of ALHS-DR}

The total sample size of LHS-DR algorithm and ALHS-DR algorithm are 1031213 and 815808 respectively. Compared with LHS-DR algorithm, the total sample size of ALHS-DR algorithm is decreased by $20.89 \%$ and the CPU time consuming is decreased by $42.52 \%$. Thus the proposed algorithm, ALHS-DR can effectively reduce the computational complexity.

\section{Decision method comparison}

Based on the properties of each traffic performance index and the objective environment, this paper defines the road capacity as benefit attribute, average vehicle stop rate and average slow mode transportation delay as cost attribute, average vehicle delay as interval attribute. The optimum average vehicle delay interval is [25.0s, 28.0s].

TABLE II. RESULTS OBTAINED BY DIFFERENT DECISION METHODS

\begin{tabular}{|l|c|c|c|}
\hline Decision methods & $\begin{array}{c}\text { Entropy- } \\
\text { TOPSIS }\end{array}$ & $\begin{array}{c}\text { Interval- } \\
\text { TOPSIS }\end{array}$ & RMODM \\
\hline Cycle (s) & 139.6718 & 97.7168 & 97.4910 \\
\hline Average vehicle delay (s/pcu) & 48.4668 & 34.8707 & 34.8306 \\
\hline Average vehicle stop rate & 0.9282 & 0.9389 & 0.9390 \\
\hline Road capacity mode & 6498.5156 & 6283.3446 & 6281.6859 \\
\hline $\begin{array}{l}\text { Average slow delay } \\
\text { transportation den }\end{array}$ & 4 & 37.1015 & 37.0632 \\
\hline Degree of robustness & 3 & 3 \\
\hline
\end{tabular}

Table II gives the performance values under different decision methods: Entropy-TOPSIS [8], Interval-TOPSIS [8], the proposed robust multi-objective optimization and decision method (RMODM). It can be seen from Table II that the control effects of Interval-TOPSIS and MODMA are similar, MODMA can bring better traffic benefit in off-line signal timing control system. Compared with the Entropy-TOPSIS, MODMA can meet the subjective and objective needs simultaneously. It decreases the average vehicle delay by $28.14 \%$ and slow mode transportation delay by $28.64 \%$ with the similar degree of robustness of timing plan. Though it slightly increases the average stop rate by 1.16 and decreases the road capacity by $3.34 \%$, as a whole, the traffic benefit of the intersection is better.

\section{Timing Plan Method Comparison}

Table III gives the performance values under different timing plan method: MODMA, HCM and ARRB.

It can be seen from Table III, with the same degree of robustness, MODMA can obtained better control effects than HCM and ARRB. Compared with HCM, though MODMA decreases the road capacity by $2.31 \%$ and increases average vehicle stop rate by $0.8 \%$, it decreases the average vehicle and slow mode transportation delay by $19.14 \%$ and $19.56 \%$ respectively. Compared with ARRB, though MODMA decreases the road capacity by $1.9 \%$ and increases the average stop rate by 0.65 , it decreases the average vehicle and slow mode transportation delay by $15.6 \%$ and $15.98 \%$ respectively. Thus, MODMA can obtain better comprehensive traffic benefits.

TABLE III. PERFORMANCE INDEX VALUES OBTAINED BY DIFFERENT TIMING METHOD

\begin{tabular}{|c|c|c|c|}
\hline Timing method & HCM & ARRB & MODMA \\
\hline Cycle (s) & 123.0235 & 117.4808 & 97.4910 \\
\hline Average vehicle delay (s/pcu) & 43.0799 & 41.2704 & 34.8306 \\
\hline Average vehicle stop rate & 0.9315 & 0.9328 & 0.9390 \\
\hline $\begin{array}{c}\text { Road capacity } \\
\text { Average slow mode transportation } \\
\text { delay }\end{array}$ & 46430.6968 & 6403.8529 & 6281.6859 \\
\hline Degree of robustness & 3 & 44.1112 & 37.0632 \\
\hline
\end{tabular}

\section{CONCLUSIONS}

This paper introduces robust multi-objective optimization and decision method to solve the signal timing problem with cycle perturbation at isolated intersection. It is observed that:

- The IDR-NSGA-II algorithm can effectively reduce the computational complexity by adaptively adjusting the sample size, and gets better solutions.

- Compared with Interval-TOPSIS and Entropy-TOPSIS, MDASOI can balance the subjective needs of decision makers and the objective evaluation information to obtain satisfactory traffic benefits.

- Compared with ARRB and HCM which were widely used in practice, the multi-objective optimization and decision method based on IDR-NSGA-II and MADSOI effectively improves the comprehensive traffic benefits in intersection.

But, this paper only analysis the efficiency of the proposed method at isolated intersection. How to use this method to solve the area signal timing problem is the future research direction.

\section{REFERENCES}

[1] Webster F V. Traffic signal setting. Road Research Technical Paper No.39 London: HMSO, 1968.

[2] Ma Yingying, Yang Xiaoguang, Zeng Ying. Multi-objective Cycle Length Optimization Model and Solution. Journal of Tongji University(Natural Science), 2009, 6: 761-765.

[3] Manual H C. HCM 2000. Washington, DC: Transportation Research Board, 2000.

[4] Xiaohong Chen. Research on signal control optimization model on urban road traffic under mixed traffic condition. Beijing Jiaotong University, 2011 (In Chinese).

[5] Deb K, Gupta H. Introducing robustness in multi-objective optimization. Evolutionary Computation, 2006, 14(4): 463-494.

[6] Barrico C, Antunes C H. Robustness analysis in multi-objective optimization using a degree of robustness concept. Evolutionary Computation, 2006. CEC 2006. IEEE Congress on. IEEE, 2006: 18871892.

[7] Jian Ma, Zhi-Ping Fan, Li-Hua Huang. A subjective and objective integrated approach to determine attribute weights. European Journal of Operational Research, 1999:397-404 